focus attention to the cADPR/Ca ${ }^{++}$-signaling system and will reveal its involvement in other cellular signal transduction processes. This information will help to predict the clinical relevance of pharmacological interventions using cADPR antagonists. Furthermore, the exact mode of action of the ADP-ribosyl cyclase, CD38, the regulation of extracellular $\mathrm{Ca}^{++}$influx, and the downstream events that lead to directed cell movement will need to be elucidated to fully appreciate the importance of this system in innate host defense.

1. Tomasz A Antibiotic resistance in Streptococcus pneumoniae. Clin. Infect. Dis. 24, 85-88 (1997) 2. Örtqvist, A. et al. Aetiology, outcome and prog- nostic factors in community-acquired pneumonia requiring hospitalisation. Eur. Respir. J. 3 1105-1113 (1990).

3. Partida-Sanchéz, S. et al. Cyclic ADP-ribose production by $\mathrm{CD} 38$ regulates intracellular calcium release, extracellular calcium influx and chemotaxis in neutrophils and is required for bacterial clearance in vivo. Nature Med. 7, 1209-1216 (2001).

4. Gao, J.-L., Lee, E.J. \& Murphy, P.M. Impaired antibacterial host defence in mice lacking the $\mathrm{N}$ formylpeptide receptor. J. Exp. Med. 189, 657-662 (1999).

5. Streb, H., Irvine, R.F., Berridge, M.J. \& Schulz, I. Release of $\mathrm{Ca}^{++}$from a non-mitochondrial intracellular store in pancreatic acinar cells by inositol1,4,5-triphosphate. Nature 306, 67-68 (1983).

6. Clapper DL, Walseth TF, Dargie PJ, Lee HC. Pyridine nucleotide metabolites stimulate calcium release from sea urchin egg microsomes desensitized to inositol triphosphate. J. Biol. Chem. 262, 9561-9568 (1987)
7. Galione, A., Lee, H.C. \& Busa, W.B. $\mathrm{Ca}^{++}$-induced $\mathrm{Ca}^{++}$release in sea urchin egg homogenates: modulation by cyclic ADP-ribose. Science 253, 1143-1146 (1991)

8. Howard, M. et al. Formation and hydrolysis of cyclic ADP-ribose catalyzed by lymphocyte antigen CD38. Science 262, 1056-1059 (1993).

9. Guse, A.H. et al. Regulation of $\mathrm{Ca}^{++}$-signaling in Tlymphocytes by the second messenger cyclic ADP-ribose. Nature 398, 70-73 (1999).

10. Novak, R., Henriques, B., Charpentier, E., Normark, S. \& Tuomanen, E. Emergence of vancomycin tolerance in Streptococcus pneumoniae. Nature 399, 590-593 (1999).

Microbiology and Tumorbiology Center

\section{Karolinska Institutet}

Stockholm, Sweden

Email: staffan.normark@smi.ki.se

\title{
Making degeneration specific
}

Polyglutamine expansions due to CAC trinucleotide repeats cause various neurodegenerative disorders, including Huntington disease, spinal and bulbar muscular atrophy and numerous forms of spinocerebellar ataxia. Studies suggest that the entry of a polyglutamine-expanded protein or peptide into the nucleus is a key step in mediating neurotoxicity for most of these diseases. However, the polyglutamine diseases have a diverse range of symptoms, often with tissue-specific patterns of toxicity specific to individual diseases. So the question remains: Does neurotoxicity in these polyglutamine diseases proceed through a common pathogenic pathway? And if so, how is the cell-specific pattern of neurotoxicity achieved?

La Spada et al. may provide a clue to solving this mystery. In the 27 September issue of Neuron, they report that transcriptional interference of cell-specific genes may be responsible for the specific pattern of degeneration seen in one type of polyglutamine disease, spinocerebellar ataxia type 7 (SCA7). SCA7 is unique among the spinocerebellar ataxias because it also causes retinal degeneration. La Spada et al. find that the protein encoded by the SCA7 gene, ataxin-7, interacts with CRX, a homeobox protein in rods and cones. CRX encodes a nuclear transcription factor containing a polyglutaminerich region, and controls the expression of several photoreceptor-specific genes, including rhodopsin and the color opsins. Moreover, the polyglutamine-expanded ataxin-7 suppresses CRX transactivation. In SCA7 mutant mice, where ataxin-7 forms nuclear aggregates (magenta, see arrows in picture) in all three nu-

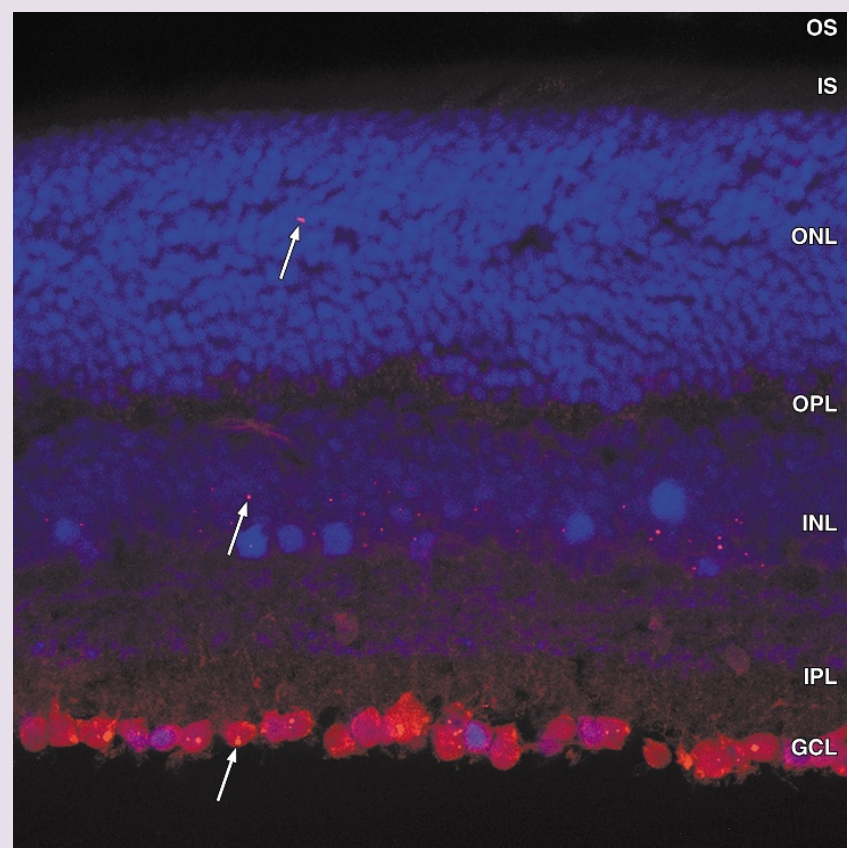

To recapitulate the cone/rod dystrophy phenotype of retinal degeneration seen in human SCA7 patients, transgenic mice expressing ataxin-7 with 92 glutamines were generated. Aggregates of ataxin-7 (magenta; marked by arrows) are seen in all 3 nuclear layers of the retina. Note that in this image, photoreceptor nuclei in the ONL and neuronal nuclei of the INL are blue, whereas nuclei in the $\mathrm{GCL}$ appear red. ONL, outer nuclear layer; OPL, outer plexiform layer; INL, inner nuclear layer; IPL, inner plexiform layer; $\mathrm{GCL}$, ganglion cell layer. Scale bar, $50 \mu \mathrm{m}$. clear layers of the retina (photoreceptor nuclei in the outer nuclear layer and neuronal nuclei in the inner nuclear layer are blue in picture, whereas the nuclei in the ganglion cell layer are colored red), the authors find that the ability of CRX to bind to its consenus sequence is diminished, and that the expression of CRX-regulated genes is reduced in the retinas of these presymptomatic mutant mice.

It would seem, from these results, that ataxin-7-mediated transcription interference of photoreceptor genes might account for the cone and rod dystrophy seen in SCA7. If generalized to other polyglutamine diseases, this suggests that transcriptional interference of a cell-type-specific transcription factor could be a common mechanism that would also produce cell-specific pathology.

KALYANi NARASIMHAN 